\title{
Improving Productivity and Control of Soil Borne Diseases of Broad Bean by Using Different Fertilization Resources under Reclaimed Soil Conditions
}

\author{
\#A. A. Abd El-Ati and Abeer M. A. El-Hadidy* \\ Plant Production Dept. and *Plant Protection Dept., Desert \\ Research Center, El-Mataria, Cairo, Egypt.
} WO FIELD experiments were conducted at El-Noubaria Province,
El Behara Governorate, Egypt during the two successive winter
seasons of $2009-2010$ and 2010-2011 to study the effect of some
organic fertilization treatments (compost, animal dung and EM
bokashi) and biological fertilization treatments \{EM1, Mycorrhiza,
Rhizobium in addition to the combination of (EM1, Mycorrhiza and
Rhizobium) \}and their interactions on damping-off and root-rot
diseases reduction in addition to enhancement of faba bean growth
and productivity under field conditions.

The organic fertilization treatments stimulated broad bean resistance to soil-borne diseases, therefore enhanced significantly broad bean growth, yield \& its attributes and chemical composition. EM bokashi, compost and animal dung surpassed the control treatment in all the studied characters. Higher significant observations were obtained from EM bokashi followed by compost and animal dung, respectively.

Applying the biological fertilization treatments decreased significantly faba bean damping off percentage and root rot incidence as well as disease severity. Consequently, increased all broad bean studied growth characters, yield and its attributes as well as its chemical composition. The higher significant observations were obtained from the combination of EM1+Mycorrhiza+Rhizobium, followed EM1, Rhizobium and Mycorrhiza, respectively.

With regard to the effect of the interaction between the organic and the biological fertilization treatments; results illustrated that all the studied growth characters, yield and its attributes, and chemical composition were increased due to the stimulation of broad bean resistance to soil-borne diseases. The uttermost values obtained from the interaction between EM bokashi $\times$ (EM1+ Mycorrhiza+ Rhizobium), followed by EM bokasi $\times$ EM1, Rhizobium then Mycorrhiza respectively. The lowermost value was obtained from control $\times$ Mycorrhiza.

Keywords: Broad bean, Productivity, Organic fertilization, Biological fertilization, EM, Bokashi, Damping off, Root-rot disease.

\footnotetext{
${ }^{\#}$ Corresponding author e-mail: dr.abdelati@gmail.com
} 
Broad bean (Vicia faba L.) is considered the world's fifth food legume after dry bean, dry pea, chickpea and lentil (Adak et al., 1998). This is due to its high nutritive value in both energy and protein contents. Therefore, increasing the plant crop production is one of the major targets of the agricultural policy in several countries.

In Egypt, faba bean is one of the main crops grown for seed; so it is an important food legume crop being the staple diet due to its high nutritive value (Nassib et al., 1991). Egypt ranked the first between the broad bean importing countries with 0.23-0.3 mega tons at the last five years. This is a result of the huge consumption, limited area for growing faba bean and its high acceptability for many diseases particularly in the new reclaimed areas such as Noubaria, Behera Governorate (Anonymous, 2011).

Soil borne diseases are considered one of the serious biotic stresses that challenge both horizontal and vertical expansion of faba bean in Egypt, particularly in the new reclaimed areas. Fungi such as Rhizoctonia solani, Fusarium solani, Sclerotium rolfsii and Macrophomina phaseolina are the most serious biotic stresses that restrict broad bean productivity causing serious losses that could reach $12 \%$ (Anonymous, 2006).

Conventional agricultural systems that embedded virtual use of chemical fertilizers and pesticides were capable to compensate both the biotic stresses and the overpopulation. Yet, health deterioration and environmental degradation and unsustainable systems are the consequences of these ways of management. Therefore, sustainable agriculture systems which embedded a wide variety of environmental practices such as bio and organic fertilization are an obligation (Mazen et al., 2008).

Organic farming systems which include compost and animal manure as fertilizers, have a lower impact to the environment than conventional production systems and could be considered as an appropriate agricultural technique. The technology of EM is an example of an appropriate technique applied into organic farming systems (Guest, 1995). EM-bokashi is an effective organic fertilizer in nature farming crop production (Kyan et al., 1999). This type of farming provides several benefits to the growers and it is an environmental friendly method of cultivation. Further, organic manures contain many plant nutrients (nitrogen, phosphorus, potassium and many other essential nutrients) and it also increases infiltration of water and enhances retention of nutrients, reduces wind and water erosion and promotes growth of beneficial organisms that help plants to resist soil borne diseases (Ross, 2008).

Correspondingly, biological fertilization is one of the acceptable procedures under organic farming regulations. Rhizobium as symbiotic bio fertilizer particularly to legumes has wide beneficial effects includes; nitrogen fixation, plant growth enhancement and inhibition of certain pathogenic fungal growth as; Macrophomina phaseolina, Rhizconia spp., Fusarium sp. and Pythium sp. Egypt. J. Agron . 35, No.2 (2013) 
(Estevez de Jensen et al., 2002; Bardin et al., 2004; Mohamedy \& Abd Allah, 2013; Mehboob et al., 2013 and Muhammad et al., 2013). Similarly, arbuscular mycorrhiza (AM) fungi are recognized as high potential agent in promoting plant growth and plant protection to several diseases caused by Phytophthora sp., Rhizoctonia sp. and Fusarium sp. (Sharma \& Doharoo, 1996; Mazen et al., 2008 and Dalpê \& Monreal, 2004).

The aim of this study is to evaluate some organic and biological fertilization procedures that are acceptable under organic farming regulations, to suppress soil borne diseases and enhance faba bean growth and productivity under field conditions.

\section{Material and Methods}

Two field experiments were conducted at $6^{\text {th }}$ October Farm - El- Noubaria province, El Behara Governorate, Egypt during the two successive winter seasons of 2009-2010 and 2010-2011 to investigate the effect of the interaction between three organic fertilization treatments (compost, animal dung and EM bokashi) and four biological fertilization treatments \{EM1, mycorrhiza, rhizobium and the combination of (EM1, Mycorrhiza and Rhizobium) \}as well as their interactions on soil borne diseases suppression and enhancement of faba bean growth and productivity under field conditions.

In regard to the organic fertilization treatments, as recommended by Kyan et al. (1999), animal manure, soil and crop residues at the ratio of 2:1:2 (w/w/w) respectively were used to prepare EM-bokashi. EM solution already prepared was sprayed onto the dry ingredients (animal manure, soil and crop residues) and mixed well until the moisture content reached within a range of $30-40 \%$. Mixture was heaped on a dry floor to a height of $15-20 \mathrm{~cm}$ and covered with a gunny bags. The temperature was maintained at $40 \pm 5^{\circ} \mathrm{C}$ during fermentation and was checked regularly. EM-bokashi was ready for use after twenty days of the preparation. Different animal manures such as cattle, poultry and goat manures were used to prepare the animal dung that used in the experiment. EM bokashi, compost which kindly obtained from the farm and animal dung was used in the rate $500 \mathrm{~kg} / \mathrm{fed}$.

Concerning the Biological fertilization treatments; EM1 consists of lactic acid bacteria, yeasts, photosynthetic bacteria, actinomycetes and mycorrhiza, was kindely obtained from the EM project- Ministry of State for Environmental Affairs, while the mycorrhiza and Rhizobium liquid cultures were kindly obtained from (Cairo MIRCEN), The Unit of Bio-fertilizers, Faculty of Agriculture - Ain Shams University- Cairo. The inoculated broad bean plants received $5 \mathrm{ml}$ of inoculum/hill just before the first irrigation (El-Mohayah). Rhizobium culture used for inoculum normally had a cell density of 10 with $10^{6}$ plant, where mycorrhiza was 60 spore/plant. Except EM they were grown in a liquid medium containing $1 \mathrm{~g} \mathrm{NH}_{4} \mathrm{CI} /$ liter (Okon et al., 1976), consequently, spores of mycorrhiza were isolated from the soil by the wet sieving and decanting method (Gerdemann \& Nicolson, 1963). 
In respect to the chemical fertilization treatment only (conventional), during soil preparation, calcium superphosphate $\left(15.5 \% \mathrm{P}_{2} \mathrm{O}_{5}\right)$ was added at the rate of 200 $\mathrm{kg} / \mathrm{fed}$, potassium sulphate $\left(48 \% \mathrm{~K}_{2} \mathrm{SO}_{4}\right)$ was added to soil just after thinning with the rate of $50 \mathrm{~kg} / \mathrm{fed}$ while nitrogen fertilization was added at two equal doses, i.e. 48 $\mathrm{kg} / \mathrm{fed}$ in the form of ammonium nitrate $(33.5 \% \mathrm{~N})$ after thinning (21 days after sowing date) and after the second irrigation (El-Mohaiah).

Soil samples were taken at depths of 30 and $60 \mathrm{~cm}$ for mechanical and chemical analysis as described by Chapman \& Pratt (1978) (Table 1).

TABLE 1. Mechanical and chemical analysis of the experimental soils at 30 and $60 \mathrm{~cm}(2009-2010$ and 2010-2011) growing seasons.

\begin{tabular}{|l|c|c|c|c|}
\hline Season & \multicolumn{2}{|c|}{$\mathbf{2 0 0 9 - 2 0 1 0}$} & \multicolumn{2}{c|}{$\mathbf{2 0 1 0 - 2 0 1 1}$} \\
\hline Depth & $\mathbf{3 0} \mathbf{~ c m}$ & $\mathbf{6 0} \mathbf{~ c m}$ & $\mathbf{3 0} \mathbf{~ c m}$ & $\mathbf{6 0} \mathbf{~ c m}$ \\
\hline \multicolumn{5}{|c|}{ Mechanical analysis } \\
\hline Sand \% & 90.8 & 94 & 91.6 & 94.4 \\
Silt \% & 3.9 & 3 & 2.96 & 3.12 \\
Clay \% & 5.3 & 3 & 5.44 & 2.48 \\
Soil texture & Sandy & Sandy & Sandy & Sandy \\
\hline Chemical analysis & 0.3 & 0.3 & 0.5 & 0.7 \\
\hline E. C. mmohs/cm ${ }^{3}$ & 11.9 & 9.75 & 10.8 & 8.8 \\
Organic matter \% & 4.1 & 4.7 & 4.2 & 4.5 \\
Soluble N ppm & 3.2 & 3.6 & 4.3 & 4.7 \\
Available P ppm & 21 & 21.3 & 24 & 23.6 \\
Exchange K ppm & \multicolumn{5}{|c}{} \\
\hline
\end{tabular}

Broad bean "faba bean" (Vicia faba L. var. Giza 2) which obtained from Agricultural Research Center (A.R.C.), Giza, Egypt was cultivated on $1^{\text {st }}$ October in both winter seasons at the rate of $60 \mathrm{~kg}$ seeds/fed. using 2-3 seeds/hill, with $25 \mathrm{~cm}$ apart in between, and was thinned to one plant/hill after three weeks from sowing date.

Strip plot design was used where the organic fertilization treatments occupied the horizontal plots and the bio- fertilization treatments arranged in the vertical plots with three replicates. The plot area was $10 \mathrm{~m}^{2}(3 \times 3.5 \mathrm{~m})$ including 5 ridges at $60 \mathrm{~cm}$ in hills $25 \mathrm{~cm}$ distance.

Weeds control was carried out after two weeks from sowing date by hand pulling and by hoeing after three weeks. However the common agricultural practices for growing faba bean were applied.

With regard to isolation and identification of the damping off and root-rot causal organisms, broad bean plants with symptoms of root-rot infection were collected in plastic bags from the field a year before the experiment and brought to the laboratory. The infected samples were rinsed in tab water and the necrotic

Egypt. J. Agron . 35, No.2 (2013) 
portions were excised and cut into at least $2 \mathrm{~mm}$, then surface sterilized with $5 \%$ sodium hypochlorite for $30 \mathrm{~s}$ and rinsed in 4 successive changes of sterile distilled water. These were then plated on potato dextrose agar (PDA) and incubated at $25 \pm 2^{\circ} \mathrm{C}$ for up to 5 days under $12 \mathrm{hr}$ photoperiod. Hyphal type transfer and the single spore technique were adopted whenever possible. Pure cultures and morphological feature were done referring to Gilman (1957), Burnett \& Hunter (1972) and Nelson et al. (1983).

The disease assessment was done periodically by examining and recording damping off after 30 and 45 days from sowing date. Percentage of root-rot disease incidence was assessed 30 days after sowing. Disease severity (D.S.) was estimated visually by assessing necrotic regions on the roots and hypocotyls using rating scale of 0-5 described according to Filion et al. (2003).

$$
\frac{\text { D.S. }=\Sigma(\mathrm{ab}) \times 100}{\mathrm{AK}}
$$

where: $a=$ No. of diseased plants having the same degree of infection. $\mathrm{b}=$ Degree of infection. $\mathrm{A}=$ Total no. of examined plants. $\mathrm{K}=$ Highest degree of infection. D.S = Disease severity.

Samples were taken from ten guarded plants per each plot after 60 days from applying the treatments to study some growth characters \{plant height $(\mathrm{cm})$, shoot fresh and dry weight $(\mathrm{g})$, root fresh and dry weight $(\mathrm{g}), 4^{\text {th }}$ leaf area $\left(\mathrm{cm}^{2}\right)$, in addition to nudes number/ plant and fresh weight of nudes per plant $(\mathrm{g})$,in addition to chemical compositions \{total pigments using Minolta SPDA-502 leaf chlorophyll meter, then converted into total chlorophyll $(\mathrm{a}+\mathrm{b})$ as $\mu$ mole $\mathrm{m}^{-2}$ referring to the equation published by John et al. (1988), total nitrogen(\%) and crude protein (\%)following the method described by Paech \& Tracey (1956), phosphorus (\%) following the method described by John (1970) and potassium (\%) referring to (Johnson \& Ulrich, 1961) \}.At harvest, yield and its components were evaluated \{No. of pods / plant, No. of seeds / pod, 100 seed weight (g) as a seed index, in addition to seed, straw and biological yields as ton/fed and No. of branches /plant .

The obtained data from the two seasons were subjected and prepared to the statistical analysis according to procedure outlined by Snedecor \& Cochran (1980) and Sehgal (2009), while Duncan's multiple range test was used to verify the significant differences between treatments means as described by Duncan (1955).

\section{Results}

Isolation of the damping off and root-rot causal organisms

The fungi isolated and identified in the sampling of the studied faba bean crop showed clear symptoms of root-rot, therefore their percentage of occurrence were recorded. In Fig. 1 it is possible to observe that $R$. solani was the dominant pathogen with $25 \%$, followed by $F$. solani with $18 \%$ then $S$. Rolfsii with $14 \%$ and M. phasolina with $10 \%$, respectively. 


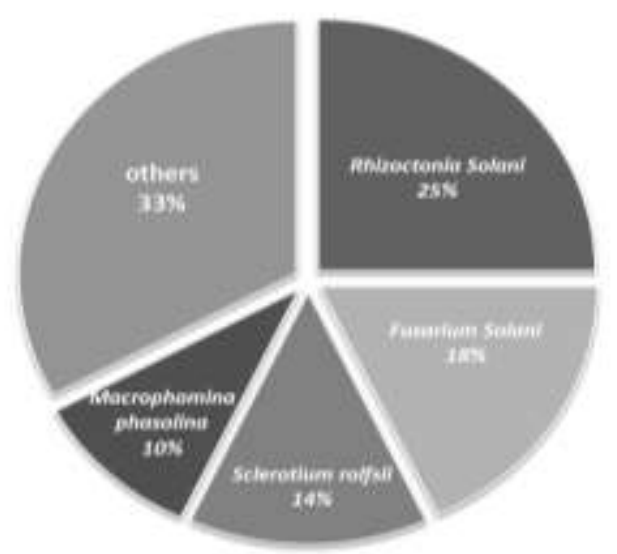

Fig. 1. Persantage of fungi isolated from faba bean roots grown in El-Noubaria destrict.

Effect of organic fertilization

Results illustrated in Tables 2,3 and 4 indicated that all faba bean studied parameters; growth characters, i.e. plant height $(\mathrm{cm})$, plant fresh and dry weight $(\mathrm{g})$, root fresh and dry weight $(\mathrm{g}), 4^{\text {th }}$ leaf area $\left(\mathrm{cm}^{2}\right)$, in addition to nudes number/ plant and nudes per plant fresh weight $(\mathrm{g})$, yield and its attributes, i.e. No. of pods per plant, No. of seeds per pod, 100 seed weight (g) as a seed index, in addition to seed, straw and biological yields (ton) per feddan and No. of branches per plant, furthermore chemical composition, i.e. total pigments, total chlorophyll content $\left(\mu \mathrm{mol} / \mathrm{m}^{2}\right)$, nitrogen, phosphorus, potassium and crude protein content \%; all increased significantly as a result of applying the organic fertilization, i.e. EM bokashi, compost, and animal dung as compared with the control treatment. The highest values were obtained from EM bokashi followed by compost then animal dung, respectively.

TABLE 2. Effect of the organic fertilization on growth characters of faba bean grown under Noubaria conditions (average of 2009/2010 and 2010/2011 growing seasons).

\begin{tabular}{|l|c|c|c|c|c|c|c|c|}
\hline \multirow{2}{*}{$\begin{array}{l}\text { Organic } \\
\text { fertilization }\end{array}$} & \multicolumn{3}{|c|}{ Shoot } & \multicolumn{2}{|c|}{ Root } & \multicolumn{2}{|c|}{ Root Nudes } \\
\cline { 2 - 6 } \cline { 5 - 8 } & $\begin{array}{c}\text { Height } \\
(\mathbf{c m})\end{array}$ & $\begin{array}{c}\text { Fresh } \\
\text { weight } \\
(\mathbf{g})\end{array}$ & $\begin{array}{c}\text { Dry } \\
\text { weight } \\
(\mathbf{g})\end{array}$ & $\begin{array}{c}\text { Fresh } \\
\text { weight } \\
(\mathbf{g})\end{array}$ & $\begin{array}{c}\text { Dry } \\
\text { weight } \\
(\mathbf{g})\end{array}$ & $\begin{array}{c}\mathbf{4}^{\text {th }} \text { Leaf } \\
\text { area( }\left(\mathbf{c m}^{2}\right)\end{array}$ & $\begin{array}{l}\text { Number/ } / \\
\text { plant }\end{array}$ & $\begin{array}{c}\text { Weight } \\
(\mathbf{g})\end{array}$ \\
\hline Without & $46.5 \mathrm{~d}$ & $33.6 \mathrm{~d}$ & $11.6 \mathrm{~d}$ & $8.5 \mathrm{~d}$ & $0.90 \mathrm{~d}$ & $2.47 \mathrm{~d}$ & $10.7 \mathrm{~d}$ & $0.13 \mathrm{~d}$ \\
\hline Compost & $57.7 \mathrm{~b}$ & $47.5 \mathrm{~b}$ & $13.7 \mathrm{~b}$ & $14.4 \mathrm{~b}$ & $2.00 \mathrm{~b}$ & $4.04 \mathrm{~b}$ & $24.4 \mathrm{~b}$ & $0.30 \mathrm{~b}$ \\
\hline Animal Dung & $53.9 \mathrm{c}$ & $41.1 \mathrm{c}$ & $12.7 \mathrm{c}$ & $12.2 \mathrm{c}$ & $1.56 \mathrm{c}$ & $3.62 \mathrm{c}$ & $18.4 \mathrm{c}$ & $0.18 \mathrm{c}$ \\
\hline EM Bokashi & $68.4 \mathrm{a}$ & $69.4 \mathrm{a}$ & $15.3 \mathrm{a}$ & $16.1 \mathrm{a}$ & $2.67 \mathrm{a}$ & $4.39 \mathrm{a}$ & $31.5 \mathrm{a}$ & $0.51 \mathrm{a}$ \\
\hline
\end{tabular}

*Means having similar letters in the same column are not statistically differed at $\mathrm{P} \geq 0.05$.

Egypt. J. Agron. 35, No.2 (2013) 
TABLE 3. Effect of the organic fertilization on yield and its attributes of faba bean grown under Noubaria conditions (average of 2009/2010 and 2010/2011 growing seasons).

\begin{tabular}{|l|c|c|c|c|c|c|c|}
\hline \multirow{2}{*}{$\begin{array}{l}\text { Organic } \\
\text { fertilization }\end{array}$} & \multirow{2}{*}{$\begin{array}{c}\text { No. } \\
\text { pods/ }\end{array}$} & \multirow{2}{*}{$\begin{array}{c}\text { No. } \\
\text { plant }\end{array}$} & seeds/ & \multirow{2}{*}{$\begin{array}{c}\text { Seed } \\
\text { pod }\end{array}$} & & \multicolumn{3}{|c|}{ Yield ton/fed } & \multirow{2}{*}{\begin{tabular}{c} 
No. \\
\cline { 5 - 7 }
\end{tabular}} \\
\cline { 5 - 7 } & & & Seed & Straw & $\begin{array}{l}\text { Biolo- } \\
\text { gical }\end{array}$ & $\begin{array}{c}\text { Branches/ } \\
\text { plant }\end{array}$ \\
\hline Without & $5.28 \mathrm{~d}$ & $2.83 \mathrm{~d}$ & $31.53 \mathrm{~d}$ & $0.76 \mathrm{~d}$ & $1.13 \mathrm{~d}$ & $1.89 \mathrm{~d}$ & $1.84 \mathrm{~d}$ \\
\hline Compost & $7.76 \mathrm{~b}$ & $3.22 \mathrm{~b}$ & $41.92 \mathrm{~b}$ & $1.25 \mathrm{~b}$ & $2.19 \mathrm{~b}$ & $3.44 \mathrm{~b}$ & $2.28 \mathrm{~b}$ \\
\hline Animal Dung & $6.40 \mathrm{c}$ & $3.07 \mathrm{c}$ & $38.90 \mathrm{c}$ & $1.08 \mathrm{c}$ & $1.88 \mathrm{c}$ & $2.96 \mathrm{c}$ & $2.15 \mathrm{c}$ \\
\hline EM Bokashi & $9.46 \mathrm{a}$ & $3.29 \mathrm{a}$ & $45.62 \mathrm{a}$ & $1.45 \mathrm{a}$ & $2.38 \mathrm{a}$ & $3.83 \mathrm{a}$ & $2.39 \mathrm{a}$ \\
\hline
\end{tabular}

*Means having similar letters in the same column are not statistically differed at $\mathrm{P} \geq 0.05$.

TABLE 4. Effect of the organic fertilization on chemical composition of faba bean grown under Noubaria conditions (average of 2009/2010 and 2010/2011 growing seasons).

\begin{tabular}{|l|c|c|c|c|c|c|}
\hline $\begin{array}{l}\text { Organic } \\
\text { fertilization }\end{array}$ & $\begin{array}{c}\text { Total } \\
\text { pigments }\end{array}$ & $\begin{array}{c}\text { Chloro- } \\
\text { phyll } \\
(\boldsymbol{\mu} \mathbf{~ m o l} \\
\mathbf{~ m}^{\mathbf{2}}\end{array}$ & $\mathbf{N} \%$ & $\mathbf{P} \%$ & $\mathbf{K} \%$ & $\begin{array}{c}\text { Crude } \\
\text { protein } \\
\text { content \% }\end{array}$ \\
\hline Without & $24.30 \mathrm{~d}$ & $227.62 \mathrm{~d}$ & $4.09 \mathrm{~d}$ & $0.80 \mathrm{~d}$ & $0.05 \mathrm{~d}$ & $25.49 \mathrm{~d}$ \\
\hline Compost & $31.13 \mathrm{~b}$ & $332.02 \mathrm{~b}$ & $4.60 \mathrm{~b}$ & $1.02 \mathrm{~b}$ & $0.08 \mathrm{~b}$ & $28.76 \mathrm{~b}$ \\
\hline $\begin{array}{l}\text { Animal } \\
\text { Dung }\end{array}$ & $28.06 \mathrm{c}$ & $282.92 \mathrm{c}$ & $4.48 \mathrm{c}$ & $0.90 \mathrm{c}$ & $0.06 \mathrm{c}$ & $27.98 \mathrm{c}$ \\
\hline EM Bokashi & $34.84 \mathrm{a}$ & $398.34 \mathrm{a}$ & $4.80 \mathrm{a}$ & $1.24 \mathrm{a}$ & $0.11 \mathrm{a}$ & $29.99 \mathrm{a}$ \\
\hline
\end{tabular}

*Means having similar letters in the same column are not statistically differed at $\mathrm{P} \geq 0.05$.

Results presented in Table 5 indicated the effect of the three organic fertilization treatments which significantly reduced pre \& post emergence damping-off and root-rot incidence compared to the control treatment (without organic fertilization). EM Bokashi was the most effective organic fertilizer that reduced both damping-off percentage by $9.69 \%$ and root rot incidence by $16.95 \%$. Disease severity as well was also reduced by organic fertilization treatments compared with the control treatment (without organic fertilization).

TABLE 5. Effect of the organic fertilization on damping off and root-rot diseases of faba bean grown under Noubaria conditions (average of 2009/2010 and 2010/2011 growing seasons).

\begin{tabular}{|l|c|c|c|c|c|}
\hline \multirow{2}{*}{$\begin{array}{l}\text { Organic } \\
\text { fertilization }\end{array}$} & \multicolumn{2}{|c|}{ Damping off \% } & \multirow{2}{*}{$\begin{array}{c}\text { Root-Rot } \\
\text { incidence \% }\end{array}$} & $\begin{array}{c}\text { Survival } \\
\text { ratio }\end{array}$ & $\begin{array}{c}\text { Disease } \\
\text { severity }\end{array}$ \\
\cline { 2 - 3 } & Pre & Post & $3.90 \mathrm{a}$ & $50.74 \mathrm{c}$ & $2.44 \mathrm{a}$ \\
\hline Without & $5.58 \mathrm{a}$ & $9.77 \mathrm{a}$ & $19.86 \mathrm{c}$ & $65.58 \mathrm{~b}$ & $1.35 \mathrm{c}$ \\
\hline Compost & $5.09 \mathrm{~b}$ & $9.26 \mathrm{~b}$ & $23.97 \mathrm{~b}$ & $63.30 \mathrm{~b}$ & $1.80 \mathrm{~b}$ \\
\hline Animal Dung & $4.87 \mathrm{c}$ & $7.87 \mathrm{c}$ & $16.59 \mathrm{~d}$ & $73.90 \mathrm{a}$ & $0.575 \mathrm{~d}$ \\
\hline EM Bokashi & $3.73 \mathrm{~d}$ & $3.70 \mathrm{~d}$ & $\mathrm{n}$ &
\end{tabular}

* Means having similar letters in the same column are not statistically differed at $\mathrm{P} \geq 0.05$. 


\section{Effect of Biological fertilization}

Data presented in Tables 6 and 7 showed that all the studied growth characters and yield and its attributes of faba bean were significantly increased as a result of the bio-fertilization treatments application. The highest significant observations of plant height, plant dry weight, root fresh weight, root nudes number/plant, root nudes weight, number of pods/ plant, number of seeds/pod and seed yield (ton/fed) were obtained from the combination of (EM1+ Mycorrhiza+ Rhizobium), followed by the chemical fertilization treatment, EM1, rhizobium and mycorrhiza, respectively. However, the plant fresh weight, root dry weight, $4^{\text {th }}$ leaf area, seed index, straw yield, biological yield and number of branches/plant; attained the highest values by using (EM1+Mycorrhiza+Rhizobium) and the chemical fertilization treatment yet, it didn't reach the significant level of 5\%, followed by significant differences between EM1, rhizobium and mycorrhiza, respectively.

TABLE 6. Effect of the biological fertilization on growth characters of faba bean grown under Noubaria conditions (average of 2009/2010 and 2010/2011 growing seasons).

\begin{tabular}{|c|c|c|c|c|c|c|c|c|}
\hline \multirow[b]{2}{*}{$\begin{array}{l}\text { Biological } \\
\text { fertilization }\end{array}$} & \multicolumn{3}{|c|}{ Shoot } & \multicolumn{2}{|c|}{ Root } & \multirow{2}{*}{$\begin{array}{c}4^{\text {th }} \text { Leaf } \\
\text { area } \\
\left(\mathrm{cm}^{2}\right)\end{array}$} & \multicolumn{2}{|c|}{ Root Nudes } \\
\hline & $\begin{array}{c}\text { Height } \\
\text { (cm) }\end{array}$ & $\begin{array}{c}\text { Fresh } \\
\text { weight } \\
\text { (g) }\end{array}$ & $\begin{array}{c}\text { Dry } \\
\text { weight } \\
\text { (g) }\end{array}$ & $\begin{array}{c}\text { Fresh } \\
\text { weight } \\
\text { (g) }\end{array}$ & $\begin{array}{c}\text { Dry } \\
\text { weight } \\
\text { (g) } \\
\end{array}$ & & $\begin{array}{c}\text { Number/ } \\
\text { plant }\end{array}$ & $\begin{array}{c}\text { Weight } \\
\text { (g) }\end{array}$ \\
\hline Chemical** & $58.7 \mathrm{~b}$ & $51.4 \mathrm{a}$ & $13.7 \mathrm{~b}$ & $13.3 \mathrm{~b}$ & $1.95 \mathrm{a}$ & $3.87 \mathrm{a}$ & $22.9 \mathrm{~b}$ & $0.31 \mathrm{~b}$ \\
\hline EM1 & $56.4 \mathrm{c}$ & $47.7 \mathrm{~b}$ & $13.3 \mathrm{c}$ & $13 \mathrm{c}$ & $1.81 \mathrm{c}$ & $3.63 \mathrm{c}$ & $21.3 \mathrm{c}$ & $0.30 \mathrm{c}$ \\
\hline Mycorrhiza & $53.4 \mathrm{e}$ & $42.2 \mathrm{~d}$ & $12.6 \mathrm{e}$ & $11.6 \mathrm{e}$ & $1.49 \mathrm{e}$ & $3.41 \mathrm{e}$ & $18.4 \mathrm{e}$ & $0.23 \mathrm{e}$ \\
\hline Rhizobium & $55 \mathrm{~d}$ & $46.3 \mathrm{c}$ & $13 \mathrm{~d}$ & $12.4 \mathrm{~d}$ & $1.66 \mathrm{~d}$ & $3.51 \mathrm{~d}$ & $19.7 \mathrm{~d}$ & $0.25 \mathrm{~d}$ \\
\hline EM1+Myco.+Rhiz. & $59.7 \mathrm{a}$ & $52 \mathrm{a}$ & $14.1 \mathrm{a}$ & $13.8 \mathrm{a}$ & $2 \mathrm{a}$ & $3.75 \mathrm{a}$ & $24.1 \mathrm{a}$ & $0.34 \mathrm{a}$ \\
\hline
\end{tabular}

*Means having similar letters in the same column are not statistically differed at $\mathrm{P} \geq 0.05$.

** The chemical fertilization with the recommended dosage as the control treatment.

TABLE 7. Effect of the biological fertilization on yield and its attributes of faba bean grown under Noubaria conditions (average of 2009/2010 and 2010/2011 growing seasons).

\begin{tabular}{|l|c|c|c|c|c|c|c|}
\hline \multirow{2}{*}{$\begin{array}{l}\text { Biological } \\
\text { fertilization }\end{array}$} & \multirow{2}{*}{$\begin{array}{c}\text { No. } \\
\text { pods/ }\end{array}$} & \multirow{2}{*}{$\begin{array}{c}\text { No. } \\
\text { seeds/ }\end{array}$} & \multirow{2}{*}{$\begin{array}{c}\text { Seed } \\
\text { index }\end{array}$} & \multicolumn{3}{|c|}{ Yield (ton/fed) } & \multirow{2}{*}{\begin{tabular}{c} 
No. \\
\cline { 5 - 7 }
\end{tabular}} \\
\cline { 5 - 7 } & plant & pod & $(g)$ & Seed & Straw & $\begin{array}{c}\text { Biolo- } \\
\text { granche/ } \\
\text { plant }\end{array}$ \\
\hline Chemical** & $7.61 \mathrm{~b}$ & $3.14 \mathrm{~b}$ & $40.91 \mathrm{a}$ & $1.24 \mathrm{~b}$ & $1.99 \mathrm{a}$ & $3.23 \mathrm{a}$ & $2.22 \mathrm{a}$ \\
\hline EM1 & $7.29 \mathrm{c}$ & $3.10 \mathrm{c}$ & $39.33 \mathrm{~b}$ & $1.18 \mathrm{c}$ & $1.91 \mathrm{~b}$ & $3.09 \mathrm{~b}$ & $2.17 \mathrm{~b}$ \\
\hline Mycorrhiza & $6.54 \mathrm{e}$ & $3.03 \mathrm{e}$ & $37.93 \mathrm{~d}$ & $1.01 \mathrm{e}$ & $1.73 \mathrm{~d}$ & $2.74 \mathrm{~d}$ & $2.07 \mathrm{~d}$ \\
\hline Rhizobium & $6.96 \mathrm{~d}$ & $3.08 \mathrm{~d}$ & $38.52 \mathrm{c}$ & $1.11 \mathrm{~d}$ & $1.85 \mathrm{c}$ & $2.96 \mathrm{c}$ & $2.11 \mathrm{c}$ \\
\hline EM1+Myco.+Rhiz. & $7.73 \mathrm{a}$ & $3.16 \mathrm{a}$ & $40.92 \mathrm{a}$ & $1.27 \mathrm{a}$ & $1.99 \mathrm{a}$ & $3.26 \mathrm{a}$ & $2.23 \mathrm{a}$ \\
\hline
\end{tabular}

*Means having similar letters in the same column are not statistically differed at $\mathrm{P} \geq 0.05$.

$* *$ The chemical fertilization with the recommended dosage as the control treatment. 
Results illustrated in Table 8 , indicated that, all the studied chemical compositions of faba bean were enhanced by the bio-fertilization application. The highest significant values of total pigments, chlorophyll $\left(\mu \mathrm{mol} / \mathrm{m}^{2}\right)$, phosphorous $\%$, crude protein $\%$, were obtained from the combination of (EM1+Mycorrhiza+Rhizobium), followed by the chemical fertilization treatment and EM1, rhizobium and mycorrhiza, respectively. As to plant nitrogen content $\%$ there was no significant differences between (EM1+Mycorrhiza+Rhizobium) and the chemical fertilization treatment. In regard to the plant potassium content $\%$, the highest significant observation was obtained from (EM1+ Mycorrhiza+ Rhizobm).

TABLE 8. Effect of the biological fertilization treatments on chemical composition of faba bean grown under Noubaria conditions (average of 2009/2010 and 2010/2011 growing seasons).

\begin{tabular}{|l|c|c|c|c|c|c|}
\hline $\begin{array}{l}\text { Biological } \\
\text { fertilization }\end{array}$ & $\begin{array}{c}\text { Total } \\
\text { Pigm- } \\
\text { ents }\end{array}$ & $\begin{array}{c}\text { Chlorophyll } \\
\left(\boldsymbol{\mu} \mathbf{~ m o l} / \mathbf{m}^{2}\right)\end{array}$ & $\mathbf{N} \%$ & $\mathbf{P} \%$ & $\mathbf{K} \%$ & $\begin{array}{c}\text { Crude } \\
\text { protein } \\
\text { content \% }\end{array}$ \\
\hline Chemical** & $30.05 \mathrm{~b}$ & $316.95 \mathrm{~b}$ & $4.58 \mathrm{a}$ & $1.03 \mathrm{~b}$ & $0.08 \mathrm{~b}$ & $28.63 \mathrm{~b}$ \\
\hline EM1 & $29.58 \mathrm{c}$ & $309.26 \mathrm{c}$ & $4.60 \mathrm{c}$ & $0.98 \mathrm{c}$ & $0.07 \mathrm{c}$ & $28.25 \mathrm{c}$ \\
\hline Mycorrhiza & $27.99 \mathrm{e}$ & $284.67 \mathrm{e}$ & $4.24 \mathrm{e}$ & $0.91 \mathrm{e}$ & $0.07 \mathrm{c}$ & $26.53 \mathrm{e}$ \\
\hline Rhizobium & $29.14 \mathrm{~d}$ & $302.46 \mathrm{~d}$ & $4.47 \mathrm{~d}$ & $0.94 \mathrm{~d}$ & $0.07 \mathrm{c}$ & $27.95 \mathrm{~d}$ \\
\hline EM1+Myco.+Rhiz. & $31.17 \mathrm{a}$ & $337.78 \mathrm{a}$ & $4.63 \mathrm{a}$ & $1.09 \mathrm{a}$ & $0.09 \mathrm{a}$ & $28.93 \mathrm{a}$ \\
\hline
\end{tabular}

*Means having similar letters in the same column are not statistically differed at $\mathrm{P} \geq 0.05$.

** The chemical fertilization with the recommended dosage as the control treatment.

Data presented in Table 9 showed that the three bio- fertilizers had significantly reduced percentage of pre and post emergence damping-off and root-rot incidence compared with the chemical fertilizer. The mixture of (EM+ Mycorrhiza+ Rhizobium) was the most effective treatment that reduced both damping-off percentage by $10.59 \%$, and root- rot incidence by $18.20 \%$. Moreover, disease severity was also reduced.

TABLE 9. Effect of the biological fertilization treatments on damping off and rootrot diseases of faba bean grown under Noubaria conditions (average of 2009/2010 and 2010/2011 growing seasons).

\begin{tabular}{|c|c|c|c|c|c|}
\hline \multirow{2}{*}{$\begin{array}{l}\text { Biological } \\
\text { fertilization }\end{array}$} & \multicolumn{2}{|c|}{ Damping off \% } & \multirow{2}{*}{$\begin{array}{c}\text { Root-Rot } \\
\text { incidence \% }\end{array}$} & \multirow{2}{*}{$\begin{array}{c}\text { Survival } \\
\text { ratio }\end{array}$} & \multirow{2}{*}{$\begin{array}{l}\text { Disease } \\
\text { severity }\end{array}$} \\
\hline & Pre & Post & & & \\
\hline Chemical** & $5.42 \mathrm{a}$ & $11.19 \mathrm{a}$ & 27.71a & $57.68 \mathrm{~d}$ & $1.91 \mathrm{a}$ \\
\hline EM1 & $4.40 \mathrm{c}$ & $7.32 \mathrm{c}$ & $19.52 \mathrm{c}$ & $68.75 b$ & $0.93 \mathrm{c}$ \\
\hline Mycorrhiza & $5.07 \mathrm{~b}$ & $7.30 \mathrm{~b}$ & $26.52 b$ & $60.37 \mathrm{c}$ & $1.76 \mathrm{~b}$ \\
\hline Rhizobium & $4.80 \mathrm{bc}$ & $8.05 \mathrm{~b}$ & $25.96 \mathrm{~b}$ & $60.93 c$ & $1.77 \mathrm{~b}$ \\
\hline EM1+Myco.+Rhiz. & $4.16 \mathrm{c}$ & $6.43 \mathrm{~d}$ & $18.20 \mathrm{~d}$ & $71.19 \mathrm{a}$ & $0.91 \mathrm{c}$ \\
\hline
\end{tabular}

*Means having similar letters in the same column are not statistically differed at $\mathrm{P} \geq 0.05$.

** The chemical fertilization with the recommended dosage as the control treatment. 
Effect of interaction between the organic and the biological fertilization

Results presented in Table 10 and 11 indicted that all the studied growth characters, i.e. plant height $(\mathrm{cm})$, plant fresh weight $(\mathrm{g})$, plant dry weight $(\mathrm{g})$, root fresh weight $(\mathrm{g})$, root dry weight $(\mathrm{g}), 4^{\text {th }}$ leaf area $\left(\mathrm{cm}^{2}\right)$, root nudes number/plant, root nudes weight $(\mathrm{g})$, as well as the yield and its attributes, i.e. No. of pods/plant, No. of seeds/pod, seed index (g), biological, seed and straw yields (ton/fed), No. of branches per plant of faba bean were promoted as a result of the interaction between the organic and the biological fertilization treatments, however mostly insignificant. As general the highest significant observations were obtained from the group of interaction between (EM Bokashi $\times$ biological fertilization), while the highest observation was obtained from \{EM Bokashi $\times$ the combination of (EM1+Mycorrhiza+Rhizobium) $\}$. The junior observations were obtained from the group of interaction between (without organic fertilization $\times$ the biological fertilization treatments).

TABLE 10. Effect of the interaction between the organic and the biological fertilization treatments on growth characters of faba bean grown under Noubaria conditions (average of 2009/2010-2010/2011 growing seasons).

\begin{tabular}{|c|c|c|c|c|c|c|c|c|c|}
\hline \multirow{2}{*}{ 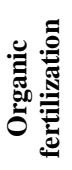 } & \multirow[b]{2}{*}{$\begin{array}{c}\text { Biological } \\
\text { fertilization }\end{array}$} & \multicolumn{3}{|c|}{ Plant } & \multicolumn{2}{|c|}{ Root } & \multirow[b]{2}{*}{$\begin{array}{c}4^{\text {th }} \text { Leaf } \\
\text { area } \\
\left(\mathrm{cm}^{2}\right)\end{array}$} & \multicolumn{2}{|c|}{ Root Nudes } \\
\hline & & $\begin{array}{c}\text { Height } \\
\text { (cm) }\end{array}$ & $\begin{array}{c}\text { Fresh } \\
\text { weight } \\
\text { (g) }\end{array}$ & \begin{tabular}{|c|} 
Dry \\
weight \\
(g)
\end{tabular} & $\begin{array}{c}\text { Fresh } \\
\text { weight } \\
\text { (g) }\end{array}$ & $\begin{array}{c}\text { Dry } \\
\text { weight } \\
\text { (g) }\end{array}$ & & $\begin{array}{c}\text { Number/ } \\
\text { plant }\end{array}$ & $\begin{array}{c}\text { Weight } \\
\text { (g) }\end{array}$ \\
\hline \multirow{5}{*}{ 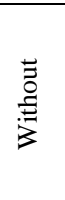 } & Chemical** & $50.7 \mathrm{k}$ & $37.4 \mathrm{jk}$ & $12.17 \mathrm{i}$ & $9.19 \mathrm{i}$ & $1.16 \mathrm{j}$ & $3.22 \mathrm{i}$ & $13 \mathrm{~m}$ & $0.14 \mathrm{hi}$ \\
\hline & EM1 & 44.91 & 33.71 & $11.89 \mathrm{ij}$ & $8.58 \mathrm{ij}$ & $0.89 \mathrm{k}$ & $2.45 \mathrm{j}$ & 10.4 no & $0.13 \mathrm{hi}$ \\
\hline & Mycorrhiza & $42.6 \mathrm{~m}$ & $29.6 \mathrm{~m}$ & $10.31 \mathrm{k}$ & $7.89 \mathrm{j}$ & 0.701 & $2.02 \mathrm{k}$ & $8.7 \mathrm{o}$ & $0.12 \mathrm{hi}$ \\
\hline & Rhizobium & $43.9 \mathrm{~lm}$ & $32.9 \mathrm{~lm}$ & $11.53 \mathrm{j}$ & $8.15 \mathrm{j}$ & $0.80 \mathrm{kl}$ & $2.19 \mathrm{k}$ & 9.50 & $0.12 \mathrm{hi}$ \\
\hline & $\begin{array}{l}\text { EM1+Myco.+ } \\
\text { Rhiz. }\end{array}$ & $50.5 \mathrm{k}$ & 34.41 & $11.93 \mathrm{i}$ & $8.93 \mathrm{i}$ & $0.96 \mathrm{k}$ & $2.49 \mathrm{j}$ & $12.1 \mathrm{mn}$ & $0.14 \mathrm{hi}$ \\
\hline \multirow{5}{*}{ 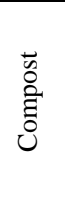 } & Chemical** & 58.5 ef & $49.2 \mathrm{ef}$ & 14.08de & $14.6 \mathrm{de}$ & $2.04 \mathrm{de}$ & $4.12 \mathrm{~cd}$ & $25.1 \mathrm{fg}$ & $0.32 \mathrm{e}$ \\
\hline & EM1 & $57.6 \mathrm{fg}$ & $46.2 \mathrm{fg}$ & $13.81 \mathrm{e}$ & $14.56 \mathrm{de}$ & 2.0def & $4.10 \mathrm{~cd}$ & $24.3 \mathrm{gh}$ & $0.28 \mathrm{f}$ \\
\hline & Mycorrhiza & $55.8 \mathrm{ghi}$ & $45.8 \mathrm{fg}$ & $13.22 \mathrm{fg}$ & $13.78 \mathrm{fg}$ & $1.92 \mathrm{ef}$ & $3.86 \mathrm{ef}$ & $22.5 \mathrm{hij}$ & $0.26 f$ \\
\hline & Rhizobium & $56.2 \mathrm{gh}$ & $46.1 \mathrm{fg}$ & $13.27 \mathrm{f}$ & $14.21 \mathrm{ef}$ & $1.97 \mathrm{def}$ & $3.97 \mathrm{de}$ & 23.4 ghi & $0.27 \mathrm{f}$ \\
\hline & $\begin{array}{l}\text { EM1+Myco.+ } \\
\text { Rhiz. }\end{array}$ & $60.5 \mathrm{e}$ & $50.4 \mathrm{e}$ & $14.24 \mathrm{~cd}$ & $15 \mathrm{cde}$ & 2.09de & $4.18 b c$ & 26.9 ef & $0.34 \mathrm{e}$ \\
\hline \multirow{5}{*}{ 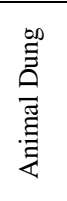 } & Chemical** & $54.2 \mathrm{hij}$ & $41.9 \mathrm{hi}$ & $12.84 \mathrm{gh}$ & $13.17 \mathrm{~g}$ & $1.71 \mathrm{gh}$ & $3.68 \mathrm{fg}$ & $20.8 \mathrm{jk}$ & $0.19 \mathrm{~g}$ \\
\hline & EM1 & $54.1 \mathrm{hij}$ & $40 \mathrm{ij}$ & $12.78 \mathrm{~h}$ & $13.17 \mathrm{~g}$ & $1.57 \mathrm{hi}$ & $3.57 \mathrm{gh}$ & $19.1 \mathrm{k}$ & $0.16 \mathrm{gh}$ \\
\hline & Mycorrhiza & $52.6 \mathrm{jk}$ & $39.3 \mathrm{ij}$ & $12.57 \mathrm{~h}$ & $9.36 \mathrm{i}$ & $1.22 \mathrm{j}$ & $3.46 \mathrm{~h}$ & $13.9 \mathrm{~m}$ & $0.15 \mathrm{ghi}$ \\
\hline & Rhizobium & $53.9 \mathrm{ij}$ & $39.2 \mathrm{ij}$ & $12.64 \mathrm{~h}$ & $11.7 \mathrm{~h}$ & $1.45 \mathrm{i}$ & $3.45 \mathrm{gh}$ & 16.51 & $0.15 \mathrm{jhi}$ \\
\hline & $\begin{array}{l}\text { EM1+Myco.+ } \\
\text { Rhiz. }\end{array}$ & 54.6 hij & $44.5 \mathrm{gh}$ & $12.87 \mathrm{gh}$ & $13.35 \mathrm{~g}$ & $1.84 \mathrm{fg}$ & $3.85 \mathrm{ef}$ & $21.7 \mathrm{ij}$ & $0.25 f$ \\
\hline \multirow{5}{*}{ 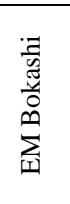 } & Chemical** & $71.2 \mathrm{a}$ & $76.8 \mathrm{a}$ & $15.82 \mathrm{~b}$ & $16.1 \mathrm{~b}$ & $2.9 \mathrm{~b}$ & $4.44 \mathrm{a}$ & $32.9 \mathrm{~b}$ & $0.59 \mathrm{~b}$ \\
\hline & EM1 & $68.9 \mathrm{~b}$ & $70.9 \mathrm{~b}$ & $14.64 \mathrm{c}$ & $15.8 \mathrm{bc}$ & $2.78 \mathrm{~b}$ & $4.40 \mathrm{a}$ & $31.2 \mathrm{bc}$ & $0.47 \mathrm{c}$ \\
\hline & \begin{tabular}{|l|} 
Mycorrhiza \\
\end{tabular} & $62.9 \mathrm{~d}$ & $54.2 \mathrm{~d}$ & $14.30 \mathrm{~cd}$ & $15.3 \mathrm{~cd}$ & $2.12 \mathrm{~d}$ & $4.30 \mathrm{abc}$ & $28.6 \mathrm{de}$ & $0.40 \mathrm{~d}$ \\
\hline & Rhizobium & $65.9 \mathrm{c}$ & $66.6 \mathrm{c}$ & $14.52 \mathrm{c}$ & $15.5 \mathrm{bc}$ & $2.44 \mathrm{c}$ & $4.34 \mathrm{ab}$ & $29.5 \mathrm{~cd}$ & $0.45 \mathrm{c}$ \\
\hline & $\begin{array}{l}\text { EM1+Myco.+ } \\
\text { Rhiz. }\end{array}$ & $73 a$ & $78.5 \mathrm{a}$ & $17.24 \mathrm{a}$ & $17.9 \mathrm{a}$ & $3.13 \mathrm{a}$ & $4.47 \mathrm{a}$ & $35.5 \mathrm{a}$ & $0.63 \mathrm{a}$ \\
\hline
\end{tabular}

*Means having similar letters in the same column are not statistically differed at $\mathrm{P} \geq 0.05$.

** The chemical fertilization with the recommended dosage as the control treatment. 
TABLE 11. Effect of the interaction between the organic and the biological fertilization treatments on yield and its attributes of faba bean grown under Noubaria conditions (average of 2009/2010-2010/2011 growing seasons).

\begin{tabular}{|c|c|c|c|c|c|c|c|c|}
\hline \multirow{2}{*}{ 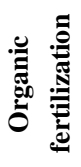 } & \multirow{2}{*}{$\begin{array}{l}\text { Biological } \\
\text { fertilization }\end{array}$} & \multirow{2}{*}{$\begin{array}{l}\text { No. } \\
\text { pods/ } \\
\text { plant }\end{array}$} & \multirow{2}{*}{$\begin{array}{c}\text { No. } \\
\text { seeds/ } \\
\text { pod }\end{array}$} & \multirow{2}{*}{$\begin{array}{c}\text { Seed } \\
\text { index } \\
(\mathrm{g})\end{array}$} & \multicolumn{3}{|c|}{ Yield ( ton/fed) } & \multirow{2}{*}{$\begin{array}{c}\text { No. } \\
\text { Branches/ } \\
\text { plant }\end{array}$} \\
\hline & & & & & Seed & Straw & $\begin{array}{l}\text { Biolo- } \\
\text { gical }\end{array}$ & \\
\hline \multirow{5}{*}{ 咅 } & Chemical** & $5.7 \mathrm{ij}$ & $2.90 \mathrm{e}$ & $35.6 \mathrm{i}$ & $0.94 \mathrm{~g}$ & $1.31 \mathrm{~h}$ & 2.251 & 2.021 \\
\hline & EM1 & $5.3 \mathrm{jk}$ & $2.90 \mathrm{e}$ & $31.3 \mathrm{j}$ & $0.81 \mathrm{~h}$ & $1.11 \mathrm{i}$ & $1.92 \mathrm{mn}$ & $1.89 \mathrm{~m}$ \\
\hline & Mycorrhiza & 4.71 & $2.60 \mathrm{~g}$ & $29.6 \mathrm{k}$ & $0.51 \mathrm{j}$ & $0.97 \mathrm{j}$ & $1.48 \mathrm{o}$ & $1.61 \mathrm{o}$ \\
\hline & Rhizobium & $5.1 \mathrm{kl}$ & $2.80 \mathrm{f}$ & $29.6 \mathrm{k}$ & $0.70 \mathrm{i}$ & $1.05 \mathrm{ij}$ & $1.75 \mathrm{n}$ & $1.70 \mathrm{n}$ \\
\hline & $\begin{array}{l}\text { EM1+Myco.+ } \\
\text { Rhiz }\end{array}$ & $5.6 \mathrm{ij}$ & $2.90 \mathrm{e}$ & $31.4 \mathrm{j}$ & $0.86 \mathrm{~h}$ & $1.23 \mathrm{~h}$ & $2.091 \mathrm{~m}$ & 2.001 \\
\hline \multirow{5}{*}{$\begin{array}{l}\overrightarrow{0} \\
0 \\
\vdots \\
0 \\
0\end{array}$} & Chemical** & $8.0 \mathrm{de}$ & $3.21 \mathrm{~b}$ & $42.6 \mathrm{~cd}$ & $1.31 \mathrm{~d}$ & $2.22 \mathrm{bcd}$ & $3.53 \mathrm{de}$ & 2.31def \\
\hline & EM1 & 7.7 ef & $3.21 \mathrm{~b}$ & $41.5 \mathrm{~d}$ & $1.23 \mathrm{e}$ & $2.22 \mathrm{bcd}$ & $3.45 \mathrm{def}$ & $2.28 \mathrm{ef}$ \\
\hline & Mycorrhiza & $7.2 \mathrm{~g}$ & $3.21 \mathrm{~b}$ & $41.2 \mathrm{de}$ & $1.18 \mathrm{ef}$ & $2.11 \mathrm{de}$ & $3.29 \mathrm{fgh}$ & $2.22 \mathrm{gh}$ \\
\hline & Rhizobium & $7.5 \mathrm{fg}$ & $3.21 \mathrm{~b}$ & $41.4 \mathrm{~d}$ & $1.20 \mathrm{e}$ & $2.14 \mathrm{~cd}$ & 3.35 efg & $2.26 \mathrm{fg}$ \\
\hline & $\begin{array}{l}\text { EM1+Myco.+ } \\
\text { Rhiz }\end{array}$ & $8.3 \mathrm{~d}$ & $3.29 \mathrm{a}$ & $43.0 \mathrm{c}$ & $1.35 \mathrm{~d}$ & $2.26 \mathrm{bc}$ & $3.61 \mathrm{~d}$ & $2.32 \mathrm{cde}$ \\
\hline \multirow{5}{*}{ 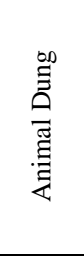 } & Chemical** & $6.5 \mathrm{~h}$ & $3.12 \mathrm{c}$ & $39.7 \mathrm{f}$ & $1.11 \mathrm{f}$ & $1.99 \mathrm{ef}$ & $3.11 \mathrm{hi}$ & $2.16 \mathrm{ij}$ \\
\hline & EM1 & $6.4 \mathrm{~h}$ & $3.00 \mathrm{~d}$ & $39.3 \mathrm{fg}$ & $1.11 \mathrm{f}$ & $1.97 \mathrm{f}$ & $3.09 \mathrm{ij}$ & $2.16 \mathrm{ij}$ \\
\hline & Mycorrhiza & $6.0 \mathrm{i}$ & $3.00 \mathrm{~d}$ & $37.7 \mathrm{~h}$ & $0.99 \mathrm{~g}$ & $1.57 \mathrm{~g}$ & $2.56 \mathrm{k}$ & $2.09 \mathrm{k}$ \\
\hline & Rhizobium & $6.4 \mathrm{~h}$ & $3.00 \mathrm{~d}$ & $37.9 \mathrm{gh}$ & $1.00 \mathrm{~g}$ & $1.89 \mathrm{f}$ & $2.89 \mathrm{j}$ & $2.12 \mathrm{jk}$ \\
\hline & $\begin{array}{l}\text { EM1+Myco.+ } \\
\text { Rhiz }\end{array}$ & $6.7 \mathrm{~h}$ & $3.12 \mathrm{c}$ & $40.0 \mathrm{ef}$ & $1.17 \mathrm{ef}$ & $2.00 \mathrm{ef}$ & 3.17ghi & $2.20 \mathrm{hi}$ \\
\hline \multirow{5}{*}{ 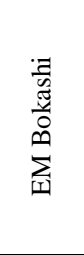 } & Chemical** & $10.2 \mathrm{a}$ & $3.29 \mathrm{a}$ & $45.9 \mathrm{~b}$ & $1.61 \mathrm{ab}$ & $2.48 \mathrm{a}$ & $4.09 \mathrm{a}$ & $2.41 \mathrm{ab}$ \\
\hline & EM1 & $9.7 \mathrm{~b}$ & $3.29 \mathrm{a}$ & $45.2 \mathrm{~b}$ & $1.54 \mathrm{bc}$ & $2.33 \mathrm{~b}$ & $3.87 \mathrm{~b}$ & $2.37 \mathrm{bc}$ \\
\hline & Mycorrhiza & $8.3 \mathrm{~d}$ & $3.29 \mathrm{a}$ & $43.2 \mathrm{c}$ & $1.38 \mathrm{~d}$ & $2.27 \mathrm{bc}$ & $3.65 \mathrm{~cd}$ & $2.36 \mathrm{bcd}$ \\
\hline & Rhizobium & $8.8 \mathrm{c}$ & $3.29 \mathrm{a}$ & $45.1 \mathrm{~b}$ & $1.52 \mathrm{c}$ & $2.31 \mathrm{~b}$ & $3.83 \mathrm{bc}$ & $2.37 \mathrm{bc}$ \\
\hline & $\begin{array}{l}\text { EM1+Myco.+ } \\
\text { Rhiz }\end{array}$ & $10.4 \mathrm{a}$ & $3.29 \mathrm{a}$ & $48.7 \mathrm{a}$ & $1.68 \mathrm{a}$ & $2.50 \mathrm{a}$ & $4.18 \mathrm{a}$ & $2.43 \mathrm{a}$ \\
\hline
\end{tabular}

*Means having similar letters in the same column are not statistically differed at $\mathrm{P} \geq 0.05$. ** The chemical fertilization with the recommended dosage as the control treatment.

Consequently, the chemical composition of faba bean was enhanced as a result of the interaction between the organic and the biological fertilization, however mostly insignificant as obtained in Table 12. Generally the highest significant observations of total pigments, chlorophyll $\left(\mu \mathrm{mol} / \mathrm{m}^{2}\right)$, total nitrogen $\%$, phosphorous $\%$, potassium $\%$ and crude protein $\%$, were obtained from the group of interaction between (EM Bokashi $\times$ biological fertilization), while the highest observation was obtained from $\{$ EM Bokashi $\times$ the combination of (EM1+ Mycorrhiza+ Rhizobium) $\}$. 
TABLE 12. Effect of the interaction between the organic and the biological fertilization treatments on chemical composition of faba bean grown under Noubaria conditions (average of 2009/2010-2010/2011 growing seasons).

\begin{tabular}{|c|c|c|c|c|c|c|c|}
\hline 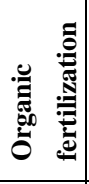 & $\begin{array}{l}\text { Biological } \\
\text { fertilization }\end{array}$ & $\begin{array}{c}\text { Total } \\
\text { Pigm- } \\
\text { ents }\end{array}$ & $\begin{array}{c}\text { Chloro- } \\
\text { phyll } \\
(\boldsymbol{\mu ~ m o l ~ / ~} \\
\left.\mathbf{m}^{2}\right) \\
\end{array}$ & $\mathbf{N} \%$ & $\mathbf{P} \%$ & K \% & $\begin{array}{c}\text { Crude } \\
\text { protein } \\
\text { content } \\
\% \\
\end{array}$ \\
\hline \multirow{5}{*}{ 咅 } & Chemical** & $25.05 \mathrm{~g}$ & $237.9 \mathrm{~h}$ & $4.30 \mathrm{i}$ & $0.87 \mathrm{ij}$ & $0.06 \mathrm{~h}$ & $26.86 \mathrm{i}$ \\
\hline & EM1 & $24.44 \mathrm{gh}$ & 249.4hi & $4.21 \mathrm{jk}$ & $0.84 \mathrm{j}$ & $0.05 \mathrm{i}$ & $26.32 \mathrm{jk}$ \\
\hline & Mycorrhiza & $23.23 \mathrm{i}$ & $212.9 \mathrm{i}$ & 3.431 & $0.72 \mathrm{k}$ & $0.04 \mathrm{j}$ & 21.451 \\
\hline & Rhizobium & $23.75 \mathrm{hi}$ & $219.9 \mathrm{i}$ & $4.18 \mathrm{k}$ & $0.72 \mathrm{k}$ & $0.05 \mathrm{i}$ & $26.11 \mathrm{k}$ \\
\hline & EM1+Myco.+Rhiz. & $25.05 \mathrm{~g}$ & $237.9 \mathrm{~h}$ & $4.27 \mathrm{ij}$ & $0.85 \mathrm{j}$ & $0.06 \mathrm{~h}$ & $26.70 \mathrm{ij}$ \\
\hline \multirow{5}{*}{$\begin{array}{l}\overrightarrow{0} \\
\text { :े } \\
\text { ठे }\end{array}$} & Chemical** & $31.37 \mathrm{de}$ & 336.0ef & 4.61defg & $1.08 \mathrm{~d}$ & $0.08 \mathrm{e}$ & $28.82 \mathrm{defg}$ \\
\hline & EM1 & $31.03 \mathrm{de}$ & $330.0 \mathrm{ef}$ & 4.60defg & $1.01 \mathrm{e}$ & $0.07 \mathrm{f}$ & 28.76defg \\
\hline & Mycorrhiza & $30.60 \mathrm{e}$ & $323 \mathrm{f}$ & $4.57 \mathrm{fg}$ & $0.95 f g$ & $0.06 \mathrm{~g}$ & $28.54 \mathrm{fg}$ \\
\hline & Rhizobium & $30.68 \mathrm{e}$ & $324.4 \mathrm{f}$ & 4.58efg & $0.97 \mathrm{ef}$ & $0.06 \mathrm{~g}$ & $28.65 \mathrm{efg}$ \\
\hline & EM1+Myco.+Rhiz. & $31.98 \mathrm{~cd}$ & 346.4de & 4.64def & $1.08 \mathrm{~d}$ & $0.08 \mathrm{e}$ & 29.03def \\
\hline \multirow{5}{*}{ 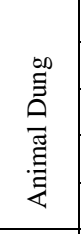 } & Chemical** & $28.94 \mathrm{f}$ & $296.3 \mathrm{~g}$ & $4.54 \mathrm{~g}$ & $0.92 \mathrm{gh}$ & $0.06 \mathrm{~g}$ & $28.38 \mathrm{~g}$ \\
\hline & EM1 & $28.51 \mathrm{f}$ & $289.5 \mathrm{~g}$ & $4.53 \mathrm{~g}$ & $0.90 \mathrm{ghi}$ & $0.06 \mathrm{~h}$ & $28.33 \mathrm{~g}$ \\
\hline & Mycorrhiza & $25.39 \mathrm{~g}$ & $242.8 \mathrm{~h}$ & $4.32 \mathrm{i}$ & $0.88 \mathrm{hij}$ & $0.06 \mathrm{~h}$ & $26.98 \mathrm{i}$ \\
\hline & Rhizobium & $28.25 \mathrm{f}$ & $285.5 \mathrm{~g}$ & $4.45 \mathrm{~g}$ & 0.90ghi & $0.06 \mathrm{~h}$ & $27.73 \mathrm{~h}$ \\
\hline & EM1+Myco.+Rhiz. & $29.21 \mathrm{f}$ & $300.5 \mathrm{~g}$ & $4.56 f g$ & $0.94 \mathrm{fg}$ & $0.07 \mathrm{~g}$ & $28.49 \mathrm{fg}$ \\
\hline \multirow{5}{*}{ 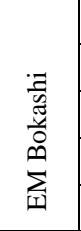 } & Chemical** & $34.84 \mathrm{~b}$ & $397.5 \mathrm{~b}$ & $4.87 \mathrm{~b}$ & $1.27 \mathrm{~b}$ & $0.11 \mathrm{~b}$ & $30.45 \mathrm{~b}$ \\
\hline & EM1 & $34.32 \mathrm{~b}$ & $387.9 \mathrm{bc}$ & $4.73 \mathrm{c}$ & $1.15 \mathrm{c}$ & $0.10 \mathrm{c}$ & $29.58 \mathrm{c}$ \\
\hline & Mycorrhiza & $32.76 \mathrm{c}$ & $360.0 \mathrm{~d}$ & 4.66cde & $1.13 \mathrm{~cd}$ & $0.08 \mathrm{e}$ & $29.15 \mathrm{cde}$ \\
\hline & Rhizobium & $33.89 \mathrm{~b}$ & $380 \mathrm{c}$ & $4.69 \mathrm{~cd}$ & $1.15 \mathrm{c}$ & $0.09 \mathrm{~d}$ & $29.30 \mathrm{~cd}$ \\
\hline & EM1+Myco.+Rhiz. & $38.39 \mathrm{a}$ & $466.3 \mathrm{a}$ & $5.03 \mathrm{a}$ & $1.48 \mathrm{a}$ & $0.13 \mathrm{a}$ & $31.47 \mathrm{a}$ \\
\hline
\end{tabular}

* Means having similar letters in the same column are not statistically differed at $\mathrm{P} \geq 0.05$.

** The chemical fertilization with the recommended dosage as the control treatment.

As indicated in Table 13 all first and second order interactions between organic and biological fertilization treatments decreased most of the studied damping- off and root-rot incidence as well disease severity. The highest reduction of damping-off and root-rot incidence in addition to disease severity were obtained from the group of interaction between (EM Bokashi $\times$ biological fertilization), while the highest observation was obtained from \{EM Bokashi $\times$ the combination of (EM1+Mycorrhiza+ Rhizobium) $\}$. 
TABLE 13. Effect of the interaction between the organic and the biological fertilization treatments on damping off and root-rot diseases of faba bean grown under Noubaria conditions (average of 2009/20102010/2011 growing seasons).

\begin{tabular}{|c|c|c|c|c|c|c|}
\hline \multirow{2}{*}{ لِّ } & \multirow{2}{*}{$\begin{array}{l}\text { Biological } \\
\text { fertilization }\end{array}$} & \multicolumn{2}{|c|}{ Damping off \% } & \multirow{2}{*}{$\begin{array}{c}\text { Root-Rot } \\
\text { incidence \% }\end{array}$} & \multirow{2}{*}{$\begin{array}{l}\text { Survival } \\
\text { ratio }\end{array}$} & \multirow{2}{*}{$\begin{array}{l}\text { Disease } \\
\text { severity }\end{array}$} \\
\hline & & Pre & Post & & & \\
\hline \multirow{5}{*}{ 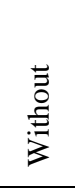 } & Chemical** & $6.24 \mathrm{a}$ & $11.09 \mathrm{~b}$ & $36.57 \mathrm{~b}$ & $46.11 \mathrm{k}$ & $2.70 \mathrm{a}$ \\
\hline & EM1 & $5.05 \mathrm{~d}$ & $9.36 \mathrm{c}$ & $33.71 \mathrm{~d}$ & $51.90 \mathrm{i}$ & $2.27 \mathrm{~b}$ \\
\hline & Mycorrhiza & $5.63 \mathrm{~b}$ & $10.48 \mathrm{bc}$ & $35.10 \mathrm{c}$ & $48.80 \mathrm{j}$ & $2.50 \mathrm{ab}$ \\
\hline & Rhizobium & $5.08 \mathrm{~d}$ & $9.06 \mathrm{c}$ & $43.58 \mathrm{a}$ & $49.48 \mathrm{ij}$ & $2.50 \mathrm{ab}$ \\
\hline & EM1+Myco.+Rhiz. & $5.03 \mathrm{~d}$ & $7.97 \mathrm{~d}$ & $29.55 \mathrm{e}$ & $57.45 \mathrm{~g}$ & $2.25 \mathrm{~b}$ \\
\hline \multirow{5}{*}{$\begin{array}{l}\overrightarrow{0} \\
\stackrel{0}{0} \\
\text { ¿े }\end{array}$} & Chemical $* *$ & $6.24 \mathrm{a}$ & $16.03 \mathrm{a}$ & $23.92 \mathrm{~g}$ & $53.81 \mathrm{hi}$ & $1.82 \mathrm{c}$ \\
\hline & EM1 & $4.52 \mathrm{e}$ & $7.19 \mathrm{~d}$ & $14.47 \mathrm{j}$ & $74.09 \mathrm{c}$ & $0 \mathrm{f}$ \\
\hline & Mycorrhiza & $5.63 \mathrm{~b}$ & $7.63 \mathrm{~d}$ & $23.57 \mathrm{~g}$ & $63.17 \mathrm{e}$ & $1.70 \mathrm{c}$ \\
\hline & Rhizobium & $5.46 \mathrm{c}$ & $9.27 \mathrm{c}$ & $23.05 \mathrm{~g}$ & 62.22ef & $1.65 \mathrm{~cd}$ \\
\hline & EM1+Myco.+Rhiz. & $3.90 \mathrm{f}$ & $6.15 \mathrm{e}$ & $14.30 \mathrm{j}$ & $75.65 \mathrm{c}$ & $0 \mathrm{f}$ \\
\hline \multirow{5}{*}{ 卺吅 } & Chemical $^{* *}$ & $5.03 \mathrm{~d}$ & $10.57 \mathrm{bc}$ & $29.29 \mathrm{e}$ & $55.11 \mathrm{~h}$ & $2.17 \mathrm{bc}$ \\
\hline & EM1 & $4.77 \mathrm{e}$ & $7.09 \mathrm{de}$ & $19.41 \mathrm{i}$ & $68.80 \mathrm{~d}$ & $1.47 \mathrm{~d}$ \\
\hline & Mycorrhiza & $5.03 \mathrm{~d}$ & $7.63 \mathrm{~d}$ & $26.52 \mathrm{f}$ & $60.82 \mathrm{f}$ & $1.90 \mathrm{c}$ \\
\hline & Rhizobium & $5.03 \mathrm{~d}$ & $7.02 \mathrm{de}$ & $25.83 \mathrm{f}$ & 62.12ef & $2.08 \mathrm{bc}$ \\
\hline & EM1+Myco.+Rhiz. & $4.51 \mathrm{e}$ & $7.02 \mathrm{de}$ & $18.81 \mathrm{i}$ & $69.66 \mathrm{~cd}$ & $1.30 \mathrm{~d}$ \\
\hline \multirow{5}{*}{ 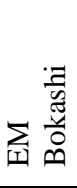 } & Chemical $* *$ & $4.16 \mathrm{e}$ & $7.10 \mathrm{de}$ & $21.06 \mathrm{gh}$ & $67.68 \mathrm{~d}$ & $1.04 \mathrm{de}$ \\
\hline & EM1 & $3.55 \mathrm{f}$ & $5.72 \mathrm{f}$ & $10.49 \mathrm{k}$ & $80.24 \mathrm{a}$ & $0 \mathrm{f}$ \\
\hline & Mycorrhiza & $3.99 \mathrm{f}$ & $6.41 \mathrm{e}$ & $20.89 \mathrm{~h}$ & $68.71 \mathrm{~d}$ & $0.95 \mathrm{e}$ \\
\hline & Rhizobium & $3.73 \mathrm{f}$ & $5.98 \mathrm{f}$ & $20.37 \mathrm{~h}$ & $69.91 \mathrm{~cd}$ & $0.87 \mathrm{e}$ \\
\hline & EM1+Myco.+Rhiz. & $3.21 \mathrm{f}$ & $4.59 \mathrm{~g}$ & $10.14 \mathrm{k}$ & $82.06 \mathrm{a}$ & $0 \mathrm{f}$ \\
\hline
\end{tabular}

*Means having similar letters in the same column are not statistically differed at $\mathrm{P} \geq 0.05$.

${ }^{* *}$ The chemical fertilization with the recommended dosage as the control treatment.

\section{Discussion}

Sustainable farming systems that imbedded certain practices which reduce the dependency on chemical fertilization and pesticides as conventional farming systems has become nowadays unpleasant for its bad consequences on environment and human health (Higa, 1993). Therefore, the essential need for alternative practices that ensure the environmental safety and sustainability has become a must. Organic farming is one of the environmental technologies that embedded several practices including organic and biological fertilization in order to provide the fertility and the resistance for a plant under biotic stress such as soil borne diseases, thus EM bokashi is a perfect example for this technology (Higa \& Parr,1994).

As obtained from the results of this study, applying the organic and biological fertilizers in addition to their interactions led to significant increase in all studied faba bean growth characters, yield and its attributes in addition to 
enhance its chemical composition similar to what reported by Javaid (2009) and Javaid \& Mahmood (2010). Moreover, the crop resistant to damping-off and root-rot diseases were also encouraged. These truths may be due to certain features in each type of fertilizer that played a very important role in its mode of action as will be described later.

Organic fertilizers are improving the soil physical, chemical and biological properties therefore, enhance water and plant relationships, thus plant growth and metabolism. In legumes; organic fertilization not only improves the soil fertility but also enhance the nodulation that suppress soil borne pathogens significantly, besides it release some organic acids to encourage the nutrients uptake by plants (Javaid \& Mahmood, 2010). Moreover, organic manures contain nitrogen, phosphorus, potassium and many other essential nutrients so it enhances retention of nutrients, consequently promotes growth of beneficial organisms that helps plants to resist soil borne diseases, thus plant growth and productivity (Ross, 2008).

Biological fertilization as well is one of the technologies that embedded in organic farming. It includes enormous number of microorganisms that provides nitrogen fixation either symbiotic or non-symbiotic, in addition to release some macro nutrients availability such as phosphorus and potassium. EM is a perfect example of these biological technologies that provides complete integration between bio-fertilization and bio-control aspects. It consists of (Phototrophic bacteria): are independent self-supporting microorganisms. These bacteria synthesize amino acids, nucleic acids, bioactive substances and sugars, substances from secretions of roots, organic matter (carbon) by using sunlight and the heat of soil as sources of energy. They can use the energy from infrared band of solar radiation from $700 \mathrm{~nm}$ to $1200 \mathrm{~nm}$ to produce the organic matter, while plants cannot, so the efficiency of the plants is increased. These metabolites are absorbed into plants directly and also act as substrates for bacteria increasing the biodiversity of the micro flora. Adding photosynthetic bacteria in the soil enhances other effective microorganisms. For example, VA (vesicular-arbuscular) mycorrhiza in the rhizosphere is increased due to the availability of nitrogenous compounds (amino acids) for use as substrates secreted by photosynthetic bacteria. VA mycorrhiza increases the solubility of phosphates in soils thereby supplying unavailable phosphorus to plants. VA mycorrhiza can coexist with Azotobactoror Rhizobium as nitrogen fixing bacteria and enhance nitrogen fixing ability of legumes. (Lactic acid bacteria): produces lactic acid from sugars. However, lactic acid is a strong sterilizer. It suppresses harmful microorganisms and increases rapid decomposition of organic matter. Moreover lactic acid bacteria enhance the breakdown of organic matter such as lignin and cellulose, and ferment these materials which normally take plenty of time. Lactic acid bacteria have the ability to suppress soil borne diseases such as Fusarium propagation which is a harmful microorganism that causes disease problem in continuous cropping. Under Fusarium conditions Egypt. J. Agron . 35, No.2 (2013) 
promotes the increase of harmful nematodes. The occurrence of nematodes disappears gradually, as lactic acid bacteria suppress the propagation and function of Fusarium. (Yeasts): synthesize antimicrobial and useful substances for plant growth from amino acids and sugars secreted by photosynthetic bacteria, organic matter and plant roots. Bioactive substances such as hormones and enzymes produced by yeasts promote active cell and root division. Their secretions are useful substrates for effective microorganisms such as lactic acid bacteria and actinomycetes. (Actinomycetes): are the structure of which is intermediate to that of bacteria and fungi, produces antimicrobial substances from amino acids secreted by photosynthetic bacteria and organic matter. These antimicrobial substances suppress harmful fungi and bacteria. Actinomycetescan coexist with photosynthetic bacteria. Thus, both species enhance the quality of the soil environment, by increasing the antimicrobial activity of the soil. (Fermenting Fungi): such as Aspergillus and Penicillium decompose organic matter rapidly to produce alcohol, esters and antimicrobial substances. These suppress odors and prevent infestation of plant pathogens. (Higa, 1993 and Anonymous, 2012).

In contrary with the EM-Bokashi, the use of the chemical fertilization under this biotic stress conditions not only affect the virgin environment and human health negatively, but it increases the probability of infection and development of root-rot pathogens as a result of plant cell elongation, rapid cell mitosis, thinness of cell walls caused by the presence of nitrogen compounds in high concentration within short time period as happened when nitrogen fertilization is applied. These conditions might beperfect for the soil borne diseases to produce its mass injury(Xu et al., 1996).

This research can provide an acceptable interpretation for the encouragement happened in faba bean growth, productivity and soil borne disease resistance when EM bokashi, EM1, Mycorrhiza and Rhizobium are integrated together to be the most appropriate organic-biological fertilization treatment under the study conditions.

\section{References}

Adak, M. S., Ulukan, H. and Guler, M. (1998) Determination of some agronomic traits in Turkish faba bean (Vicia faba L.) FABIS, News Letter, 42, 29-31.

Anonymous (2006) Furfural chemical documents. Fact sheets on new active ingredients. US Environmental Protection Agency. (http://www.epa. gov/opprd 001/fact sheets/ furfural. Pdf) $4 \mathrm{pp}$

Anonymous (2011) Egyptian Agricultural Statistics, part one: The winter crops (2009 2010). Egyptian Central Agency for Public Mobilization and Statistics. 
Anonymous (2012) Asian-Pacific Natural Agriculture; http://www. royalgcol.ac. uk/research/conferences/tabo.htm.

Bardin, S.D., Huang, H.C., Pinto, J., Amunden, E.J. and Erickson, R.S. (2004) Biological control of Pythium damping -off of pea and sugar beet by Rhizopium leguminosarium bv. Viceae. Can.J. Bot.82, 291-296.

Burnett, H.L. and Hunter, B.B. (1972) "Illustrated Genera of Imperfect Fungi” Burgess Pub. Co. Minneapolis, Minnesota, USA. 241 pp.

Chapman, H.D. and Pratt, P.F. (1978) "Methods of Analysis for Soils, Plant and Waters". University of Callifornia Division Agriculture Science. Priced publication, 4034: 50 and 169.

Dalpê, Y. and Monreal, M. (2004) Arbuscular mycorrhiza inoculum to support sustainable cropping systems.Crop Mang. pp: 301-309.

Duncan, D.B. (1955) Multiple range and multiple F-test biometrics, 11,1-24.

Estevez, De, Jensen, C., Percich, J. A. and Graha, P. H. (2002) The effect of Bacillus subtilus and Rhizobium inoculation of dry bean seed on root-rot severity and yield in Minnesola. Annu. Rep. Bean Improv. Crop. 45, 98-99.

Filion, M., St- Arnaul, M. and J abaji- Hare, S.H. (2003) Quanification of Fusarium solani f. sp. phasolina in mycorrhizal bean plants and surrounding mycorrhizosphere soil using real time polymerase chain reaction and direct isolations on selective media. Phytopathology, 93, 229-235.

Gerdemann, J.W. and Nicolson, T.H. (1963) Spors of Mycorrhizal endogen species extracted from soil by wet sieving and decanting. Trans Br. Mycol. Soc. 46, 235-244.

Gilman, C.J. (1957) “A manual of Soil Fungi”. $2^{\text {nd }}$ ed. Iowa.State Coliege Press.USA. pp. 450.

Guest, D. (1995) Bokashi (EM) as a bio-control agent to suppress the growth of Phytophthora cinnamomi: In Proceedings of the Fifth International Conference on Kyusei Nature Farming. Sangakkara U.R. and Senanayake, D. (Editors) Bangkok, Thailand. pp. 216-218.

Higa, T. (1993) Effective Microorganisms: Their Role in Kyusei Nautre Farming. Paper Presented at the $3^{\text {rd }}$ International Conference on Kyusei Nature Farming. USA. pp. 20-23.

Higa, T. and Parr, J.F. (1994) Beneficial and Effective Microorganisms for a Sustainable Agriculture and Enviroment. p. 16.

Javaid, A. (2009) Growth, nodulation and yield response of balck gram (Vignamungo L.) as influenced by biofertilizers and soil amandements. Afr. J. Biotechnol. 8 (21), 57115717.

Javaid, A. and Mahmood,N. (2010) Growth, nodulation and yield response of soybean to bio fertilizers and organic manures. P. J. Bot. 42 (2), 863-871.

Egypt. J. Agron . 35, No.2 (2013) 
John, M., Osterman, J. C. and Mitchell, J. L. (1988) Calibration of the Minolta SPDA502 leaf chlorophyll meter. Photosynthesis Research, 48, 467-472.

John, M. K. (1970) Colorimetric determination of phosphorous in soil and plant materials with ascorbic acid. Soil Sci. 109, 214-220.

Johnson, C. M. and Ulrich, A. (1961) Analytical methods for use in plant analysis. U.S. Dept. Agric., Calif. Univ. Agric., Inform. Bull. p. 766.

Kyan, T., Shintani, M., Kanda, S., Sakurai, M., Ohashi, H., Fujisawa, A. and Pongdit, S. (1999) Kyusei nature farming and the technology of effective microorganisms. International Nature Farming Research Center (INFRC), Atami, Japan and Asia Pacific Natural Agriculture Network (APNAN), Bangkok, Thailand.

Mazen, M. M., El-Batanony, Nadia H., Abd El- Monium, M.M. and Massoud, O.N. (2008) Cultural filtrate of Rhizobium spp. and arbuscular mycorrhiza are potential biological control agents against root-rot fungal diseases of faba bean. Global $\mathrm{J}$. Biotech and Biochem. 3, 32-41.

Mehboob, L., Naveed, M., Zahir, Z. and Sessitsch, A. (2013) Potential of rhizosphere bacteria for improving rhizobium-legume symbiosis. Plant Microbe Symbiosis: Fundamentals and Advances. pp. 305-349.

Mohamedy, R. S. R. and Abd Allah, M. A. (2013) Bio-priming seed treatment for biological control of soil borne fungi causing root-rot green bean (Phasealus vulgaris L.). Journal of Agricultural Technology, 9 (3), 589-599.

Muhammad Nasir Subhani, Shahbaz Talib Sahi, Liaqat Ali, Safdar Hussain, Javaid Iqbel and Nisar Hussain (2013) Management of chickpea wilt caused by Fusarium oxysporum f. sp. ciceris through antagonistic microorganisms. Canidian Journal of Plant Protection, 1(1) 1-6.

Nassib, A.M., Khalil, S.A., El-Borai, M.A. and Radi, M.M. (1991) In: "Nile Valley Regional Program on Cool Season Food Legumes and Cereals". Annual Report, Egypt: ARC/ICARDA.

Nelson, P.E., Toussoum, T.A. and Marasas, W.F. (1983) Fusarium spp. an illustrated manual for identification .The Pennsylvania University, Parl., USA. pp. 198.

Okon, Y., Alberecht, S.L. and Burries, R.H. (1976) Fixation of nitrogen by Azospirillum nlipoferum alone and with plants. Plant Physiol. 1976 supplement, $70 \mathrm{pp}$.

Paech, K. and Tracey, M. V. (1956) In "Modern Methods of Plant Analysis" SpringerVerlag, Berlin, 1 (4), 643-646.

Ross, H.M. (2008) "Managing Livestock Manure". Published by the Alberta Agriculture and Rural Development, Alberta, Available online at http//www1.agric.gov.ab.ca/ \$department/ deptdocs. nsf. 
Sehgal, D. K. (2009) Split plot and strip plot designs. In: "A Diagnostic Study of Design and Analysis of Field Experiments". pp. 377-388.

Sharma, S. and Doharoo, N. B. (1996) Vesicular-arbuscular mycorrhiza in plant heath and disease management. Intl. J. Trop. Plant Dis. 14,147-155.

Snedecor, G.W. and Cochran,W.G. (1980) “Statistical Methods" $8^{\text {th }}$ ed. Iowa State Univ. Press, Ames, Iowa, USA.

Xu, H. L., Wang, R., Mridha, M. A. U. and Umemura, U. (1996) Phytophthora resistance of tomato plants grown with EM-Bokashi. In: An earth saving revaluation (Terio Higaed.). Sunmark publishing Inc., Shinjuku-Ku , Tokyo, Japan.

(Received 11/11/2013;

accepted 11 / 2/2014) 


\section{تحسين إنتاجية ومقاومة الفول البلدى للأمراض الكامنة بالتربة

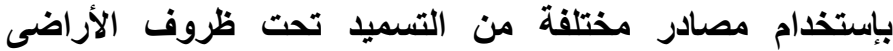 المستصلحة \\ أحمد عبد العاطي احمد وعبيز المرسي أحمد الحديدي*

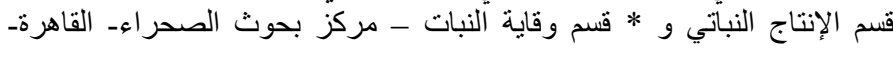

أجريت تجربتين حقليتين فى منطقة النوبارية بمحافظة البحيرة خلال الموسميين

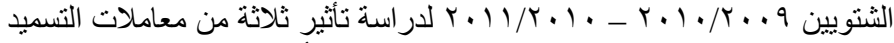

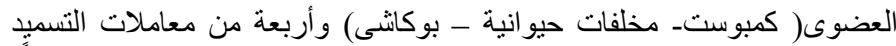

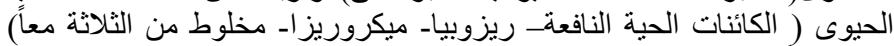

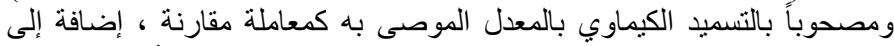

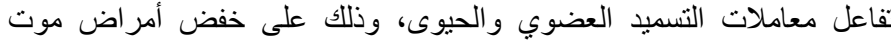

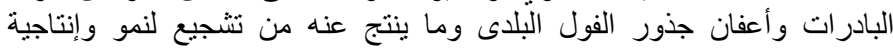

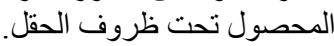

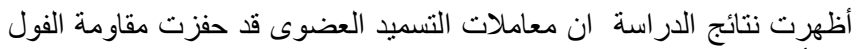

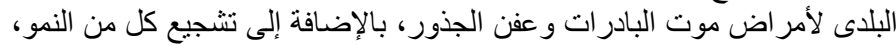

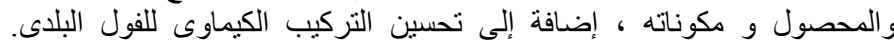

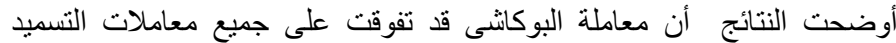

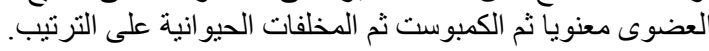

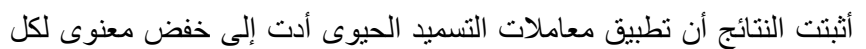

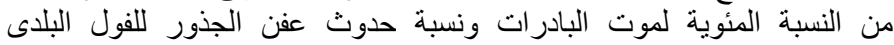

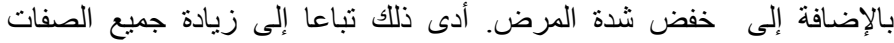

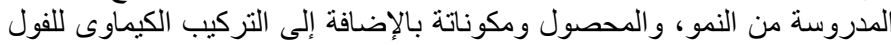

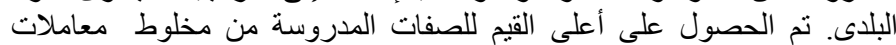

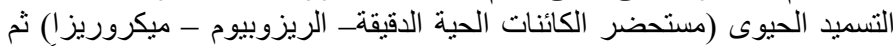

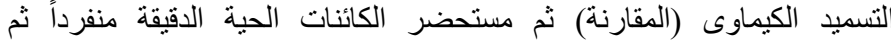
الريزوبيوم ثم الميكروريزا على الترنيب.

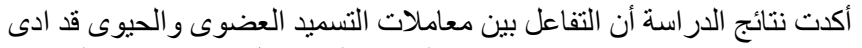

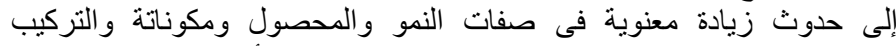

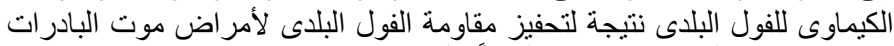

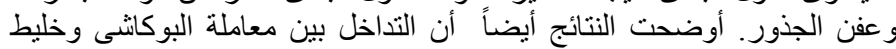

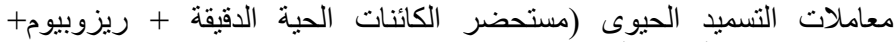

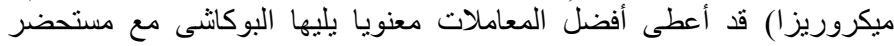

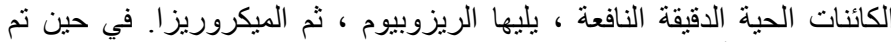

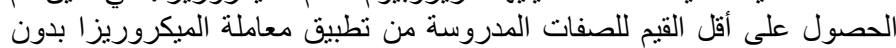

تسمبيد عضوى.

Egypt. J. Agron . 35, No.2 (2013) 
